\title{
An Energy-Efficient Duty-Cycled Wake-Up Radio Protocol for Avoiding Eavesdrop in Wireless Sensor Networks
}

\author{
P. Uma Maheswari., R. Arun Prasath
}

\begin{abstract}
Wake-Up Radio System (WuR)) is frequently regarded due to the fact the best opportunity to supplant MAC (Media get right of entry to manipulate) conventions with the commonplace cyclic cycle of chipping away at wireless sensor networks (WSN). The Double Radio Protocol (DoRa) is some other MAC conference for the WuR in-band framework with addressability. No matter the reality that the DoRa conference improves the electricity skills of the WSN, irrespective of everything it testimonies a assembly difficulty whilst the WuR framework is wanted all the time. The WuR squanders a number of vitality while it hears arousing request from superb hubs, but it isn't predicted or described in fantastic employments. On this paintings, a cyclic flexible DoRa (DC-DoRa) cycle is proposed to take care of the difficulty of listening to. The principle idea of the interest is to empower the WuR embody earlier than guidance the hub and cripple WuR after the hub has sent the statistics. Nitty gritty recreations are completed beneath OMNeT ++ utilising actual information parameters to expose the huge energy reserve price range performed with the useful resource of the two conventions and the digital disposal of over-tuning in with $D C$ DoRa. Reality be cautioned, everyday electricity utilization is a couple of instances lower with the DoRa convention contrasted with general MAC conventions. Notwithstanding the reality that the communicate can constitute as much as $93 \%$ of WuR's capacity usage with the DoRa convention, it's miles dwindled to truely $1 \%$ with the DC-DoRa convention.
\end{abstract}

Keywords - wireless Sensor Networks, Wake-Up Radio, MAC Protocol, power performance, obligation cycling.

\section{PRESENTATION}

A long way flung Sensor Networks (WSNs) are one of numerous packages, as an example, natural, contemporaryday or recovery checking. They are additionally a number one phase within the growing subject of the internet of factors [1]. Power productivity is pivotal in WSNs because sensors hubs are commonly fueled via confined batteries, whilst their substitution isn't feasible after intake. Some strategies have been considered within the writing to decrease the strength utilization of WSNs [2] [3], the difficulty being dealt with in diverse viewpoints.

The degree of vitality wolfed due to radio correspondence is frequently massive contrasted with first-rate segments, for example, Microcontroller Unit (MCU) or sensor unit. The

Revised Manuscript Received on September 14, 2019.

P. Uma Maheswari., Assistant Professor, Department of Computer Applications, Anna University Regional Campus - Madurai, Keelakuilkudi, Madurai, Tamilnadu, India (E-mail: dharshukiran@gmail.com)

R. Arun Prasath, Professor, Department of ECE, Siddhartha Institute of Technology \& Sciences, Narapally, Hyderabad, Telangana, India. (Email: prasath2k6@gmail.com) the most encouraging improvements and they're applied in

essential driving force of energy squander for a long way flung correspondence are inert tuning in and catching. Inert listening alludes to the reality of tuning in to the channel while there may be no continuous faraway transmission, and catching alludes to a hub tuning in to far flung transmissions routed to a few different hub. One-of-a-kind Medium get access to control (MAC) conventions were proposed to enhance the power effectiveness of the far off correspondence [4]. A evaluation with maximum easy MAC conventions is displayed in [2] with the accompanying characterization: TDMA-based, dispute based, and 1/2 and half of of conventions. Dispute based totally conventions are the maximum acquired methodologies with B-MAC [5] and S-MAC [6] being the maximum famous MAC conventions. Those MAC conventions essentially rely upon a low obligation cycle to decrease the hubs energy utilization. In obligation biking structures, the radio is exchanged into rest mode while there may be no correspondence as a manner to stay far from inert tuning in. All matters taken into consideration, inactive listening cannot be completely smothered with responsibility biking gadgets whilst you keep in mind that hubs want to test for doable correspondence at the same time as awakening.

Wake-up radio (WuR) frameworks were proposed at some stage inside the maximum contemporary decade to attend to the problem of inactive pay attention-ing, by using empowering the hubs to wake up on request via a particular radio message, mainly a aa003e33992aa1e42449a037e2560bf2 (WuC). Regarding the tool set-up, a 2 nd radio module is normally associated with the hubs for the wake-up correspondences and the primary radio module is utilized for information transmission. The following radio, in any other case referred to as wake-up radio, have to be a low-control device or absolutely aloof. The primary WuR configuration have become proposed in [7] with an essential radio-activated circuit. A few Wake-up Receivers (WuRx) had been proposed inside the writing to lower the prevailing usage of the WuR circuit, and they are characterised in severa sorts, for example, RFID, heterodyne, MCU or correlator [8].

Anyhow, distinct MAC conventions should be characterised for executing the WuR highlights because of the truth that they infer a correspondence with radios. Scarcely any MAC conventions were proposed at some 

WIRELESS SENSOR NETWORKS

degree inside the maximum current years to sign up for the WuR highlights, as an instance, RTM [9], On demand MAC [10] and SCM-MAC [8]. Those conventions altogether reduce the energy utilization of the hubs, however the truth that a more energy proficient wake-up plan may be done. The precept paintings looking at WuR conventions execution among themselves grow to be completed by way of [11], however it turn out to be implemented for implantable body sensor structures and reenactments were performed in MATLAB with barely any insights concerning the usage. Even as looking at these conventions is in particular captivating, it is not absolutely compelling due to the fact each WuR convention is supposed for a specific gadget. The creators in [12] as of late analyzed the presentation in their WuR stage to the maximum commonly applied MAC conventions for WSNs reenactments below 3 certifiable system preparations. Whilst inert tuning in and catching are alluded in their paper, they failed to deliver any insights concerning their effect on electricity utilization.

MAC conventions are proposed in this paper for looking after the WuRx usefulness, particularly the Double Ra-dio (DoRa) conference and responsibility-Cycled Double Radio (DC-DoRa) conference. Whilst the conventions proper usage lie inside the WuRx, they're additionally accountable for awakening the precept radio for data transmission. DCDoRa is virtually an enhance of DoRa for keeping away from the catching trouble identified with WuR frameworks. The DoRa expert tocol execution is classed with the resource of strategies for a signal Detection idea (SDT), that's then applied for assessing the vitality repartition because of catching and expected wake-up. Within the opposite of WuR conventions of the writing, the DoRa convention considers the way that dynamic hubs impart at the same time as one in every of a kind hubs are resting. This correspondence plot empowers the non-usage of RTS/CTS devices or affirmations (ACK), and in this way decreases each the vitality price and dormancy of correspondence. The DoRa and DC-DoRa conventions are finished in OMNeT++ 4.6 with the MiXiM 2.3 gadget [13] [14], with the DoRa utilization subtleties given in [15]. The reenactment system offers reproducible and predictable results with enter parameters recognized with energy usage have been given by using trial estimations.

Regardless of the reality that this paper is predominantly focused round WuR conference, we supply a concise united states about WuR device. A few WuRx fashions were proposed as of past due and a rundown of them were scientifically concept about by way of way of [16]. On the identical time as our WuRx version may be much like [17] [18], the energy usage of their MCU chargeable for the vicinity interpreting is not all around researched. For our scenario, the MCU manipulate usage and deferral have been tentatively expected on the equal time as resting or decoding a location and those measures are utilized within the take a look at system.

The paper intends to give a solution for the WuR catching hassle. Our principle dedication is to provide stable records about the strength squander because of catching while at the equal time utilizing a normally conventional (DoRa) wakeup ra-dio convention. At that factor we recommend every different MAC conference, to be particular DC-DoRa, to preserve a strategic distance from the catching problem thru essentially obligation biking the WuRx. As a long way as we must in all likelihood apprehend, there's no one of a kind paintings managing this approach.

The remainder of this paper is composed as pursues. The DoRa framework configuration is given in phase 2, wherein the sensor hub and the DoRa convention are displayed. In segment three, we dissect the exhibition of the DoRa conference by means of way of contrasting and commonplace MAC conventions and indicating the catching trouble. The brand new DC-DoRa convention is proposed in phase 4 for settling the catching problem along its exhibition investigation. At prolonged last, section 5 closes the art work and offers some factors of view.

\section{II . DORA TOOL DESIGN \& METHODOLOGY}

WuR conventions aren't absolutely characterised with out thinking about a particular machine. As there's no WuRx popular or typically recounted degree proper now, we first offer a concise portrayal of the hub and WuRx plan in this segment. The DoRa conference is then clarified.

\subsection{DoRa Node}

A few gadget modifications are required on the sensor hubs to empower the wake-up method, and all the more explicitly to be agreeable with the DoRa convention. The hub structure is portrayed in parent 1 , a semi-inactive wakeup radio collector (WuRx) that can accumulate power from had been given indicators is associated with both number one radio and precept MCU. These two segments may be isolated devices or they will be included in a unmarried device as a far off bit, as an example, TelosB, Xbee or Waspmote. Them can be arouse up thru the WuRx via an intervene on sign.

Our semi-aloof WuRx was first offered in [19] and it's far portrayed in discern 2 with the three number one segments. The detached radio beneficiary is a discounted rectenna cluster which could harvests electricity from got signals at 2.Forty five GHz. As dynamic thing, a PIC24F16KA102 from Microchip is applied as MCU due to its instead lowmanage utilization. The MCU item is basically to manner the area decoding functionality. The alternative dynamic part is a nano-manage comparator from Texas gadgets it really is applied for increasing the WuRx affectability. The semi-aloof WuRx configuration is relevant with comparative segments as long as they satisfy the 2 after situations: 1) the indifferent radio beneficiary proselytes the RF sign into a DC signal and a couple of) the MCU is a lowmanage system.

\subsection{DoRa Protocol}

The DoRa convention is supposed for severa leveled structures made from a Base Station (BS) and $\mathrm{N}$ hubs, in which a solitary bounce correspondence is applied among the BS and hubs. 
Figure 1. Node architecture with an additional wakeup radio circuit.

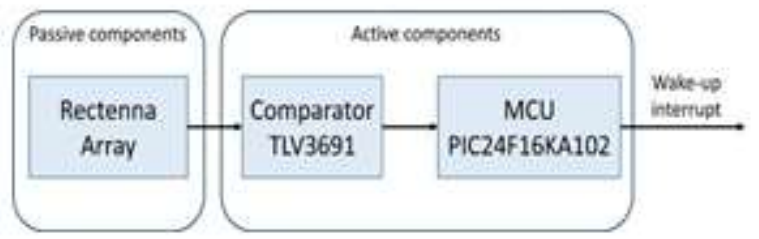

Figure 2. Our semi-passive WuRx design with specific components.

Within the course of the creation degree, every one of the hubs are placed into relaxation mode with the primary radio card they will be killed. BS sends a reminder intermittently to each hub with the useful resource of moving into the intention MAC address inside the message a reminder. On the point at the same time as a hub approve the approach arise, be dynamic and react by way of sending an statistics message legitimately to BS. The subsequent region form is portrayed in discern 3. Prior to sending every different reminder, keeping up BS sooner or later of a foreordained window Tout time restrict or till the collection of records from the hub being cited. Every hub is incited with this correspondence conspire and no interior obstruction is probably because of the hubs remain in relaxation mode at the same time as they did not get a reminder is routed to them. Correspondence tenet is printed in figure 4.

This conference infers that BS shift back and forth amongst transmission and gathering modes. WUC gathering empowered nation as soon as transported and initiated at some factor point the transmission kingdom WUC must be sent. Regarding hub, the precept radio must transfer between rest mode and the transmission mode, the closing is initiated in the wake of waking radio has affirmed reminder. Precept standards ought to meet to verify the reminder in our plan. Inside the first place, the got depth of the signal should be sufficient for a wake-up the term of the preface, as consistent with the regular voltage conveyed with the aid of indifferent wake-up circuit. The following condition is the approval of the MAC address, that is remembered for the message reminder.
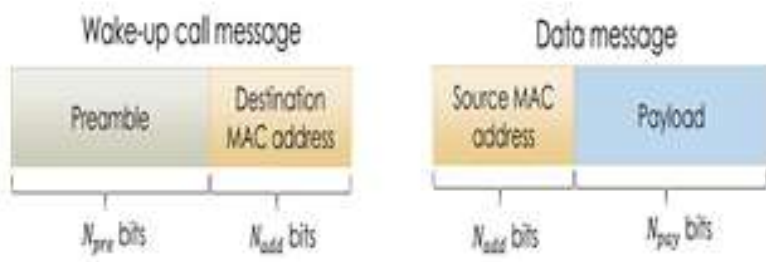

Figure3. Frame structure of exchanged messages between the BS and nodes.
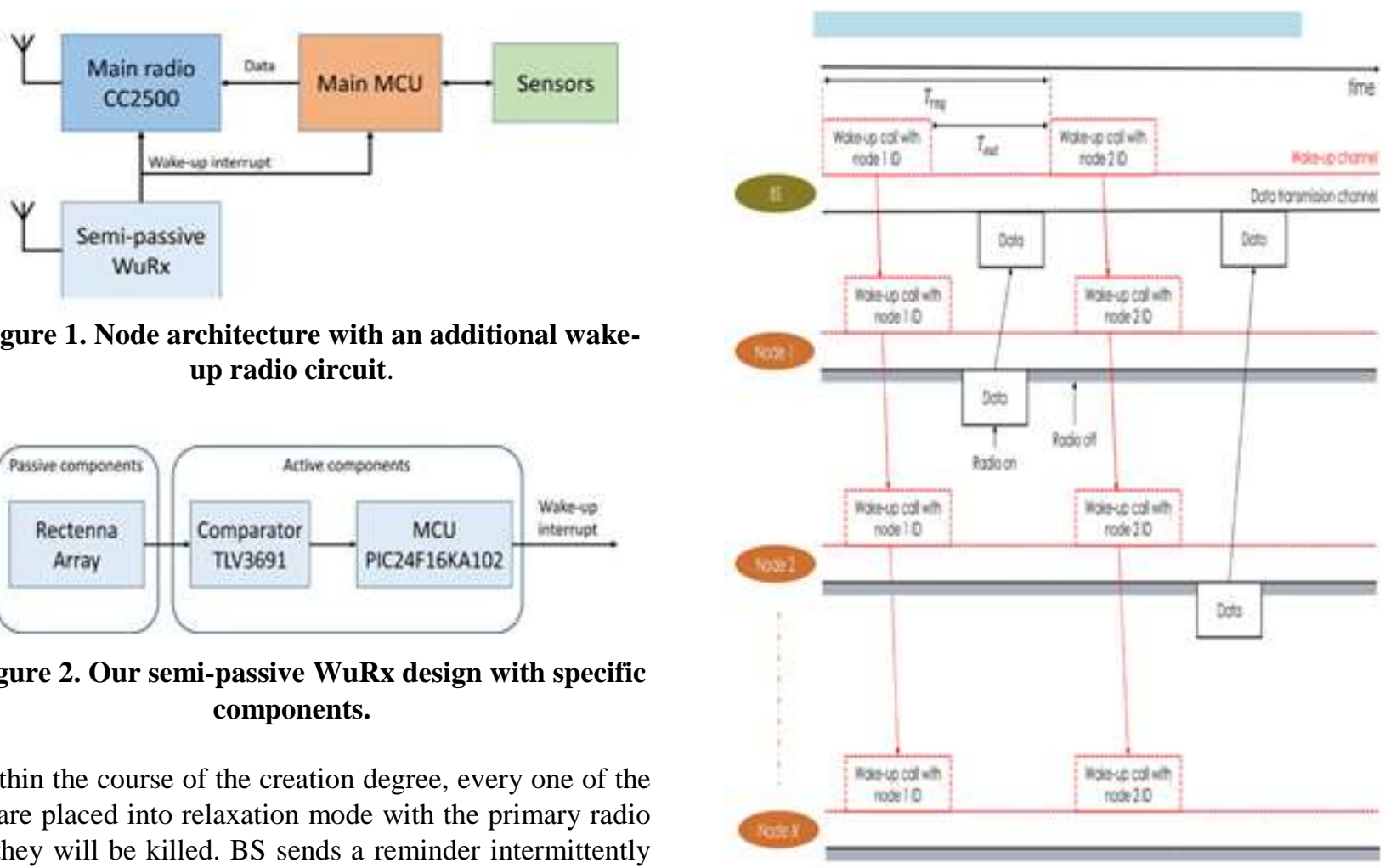

Figure 4. Communication principle with the DoRa protocol.

\section{DORA PERFORMANCE ANALYSIS}

On this segment, Dora conference execution come to be assessed in views. Preliminary, an examination with the opposite two stated MAC convention is all round served to show off the benefits of the usage of the convention DORA contrasted and the maximum widely utilized technique in WSNs today. At that factor, repartition the energy because of the correspondence added whilst using the convention Dora, which features the trouble WUR catches. Prior to 2 of this assessment, the identification sign is introduced as applied for execution studies.

\section{Three.1. Sign Detection version}

Sign Detection principle (SDT) may be applied in numerous regions for breaking down the statistics had been given from the preliminary [20]. In this paintings, the presentation of radio wake discovered through SDT via searching the radio desire awaken in the sight of a signal. Version SDT first exhibited related to radio plot wake.

Every sign is offered inside the channel and on the location of the hub is felt with the aid of WUR, which should pick whether or now not or not the signal is WUC. On the off threat that the WUC is outstanding, at that point the aim address is contrasted with the hub deal with with choose whether or not or no longer the approach is authorized wake. Activities may additionally additionally arise even as a sign is available is regarded in determine five, with the because of this of the accompanying sports:

- DS: a fulfillment area of message WUC, at the identical time as it's miles virtually present in the channel,

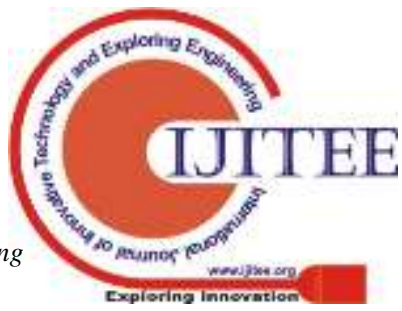



WIRELESS SENSOR NETWORKS

- DF: Failure Detection WUC messages, whilst it is surely present in the channel,

- FA: false alert, while the wake-up signal is recognized

- CR: Refusal proper, while the sign is neglected in mild of the truth that it isn't always viewed as a wake-up sign, at the same time as the WUC message is absent within the channel,

- WV: Wake-up Validation, whilst the purpose deal with (it is remembered for the message WUC) pertains to a hub cope with,

-WR: Wake-up refusal, at the same time as the purpose address you entered does not examine to the hub address or has been debased by using way of the channel.

Every event occurs with danger (A), with A being the radio preference wake recorded previously. Now not those probabilities are unfastened in mild of the truth that there are corresponding events, which can be communicated thru state of affairs fol-lowing:

$$
\begin{aligned}
& (\mathrm{DS})+(\mathrm{DF})=1 \\
& (\mathrm{FA})+(\mathrm{CR})=1 \\
& \mathrm{DS}(\mathrm{WV})+\mathrm{DS}(\mathrm{WR})=1
\end{aligned}
$$

Conditional probabilities are used in Equation (3), since the events of wake-up validation or rejection (WV or VR) occur only after a wake-up call detection ( DS ). According to Equations (1)-(3), calculating three probabilities among all is sufficient to fully analyse the system. We consider to focus on (DS), (FA) and DS (WV ), since they are more significant and suitable for calculation. A perfect wake-up radio system is cha racterized by a Detection Success probability ( DS ) equal to 1, a False Alarm probability (FA ) equal to 0 and a Wake-up Validation probability DS (WV ) equal to $1 / \mathrm{N}$, with $\mathrm{N}$ being the number of nodes.

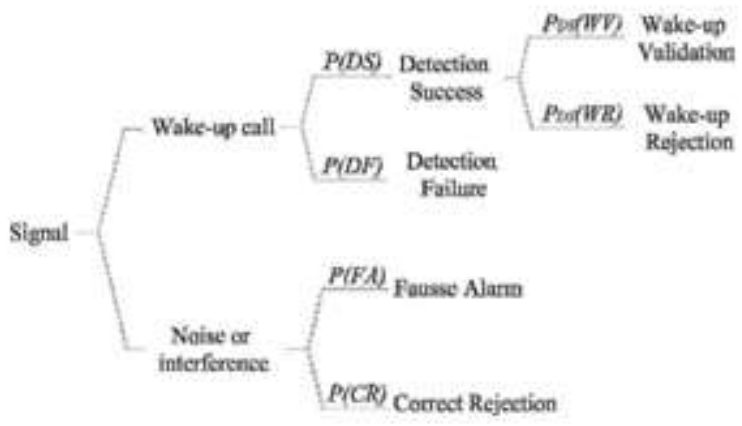

Figure 5. Tree of possible events in the presence of a signal.

\subsection{Performance Comparison with Other MAC Protocols}

Dora conference contrasted with the B-MAC [5] and IEEE 802.15.Four [21] conventions via a similar state of affairs recreations. No matter whether or not the B-MAC and IEEE 802.15.Four have been selected for execution in MiXiM, offers an interesting correlation of our convention with the wake-up on-request way to address duty cycle (BMAC) and continuously on manner to cope with radio (IEEE 802.15.Four).

A system contains of a BS and forty hubs conveyed haphazardly in the vicinity of four hundred $\mathrm{m} 2$ is the BS, with direct transmission. Between bundle at the same time as it is absent inside the channel, characterized and each hub intermittently transmit facts to

appearance time (IPAT) is de-fined because the time most of the receipt of two successive bundles in BS and it portrays the tempo of traffic. Kinds IPAT allow evaluation of device execution for each convention underneath severa visitors load, that is dictated by using the utility scenario. Different interest parameters are set to a steady worth and the number one parameters are given in table 1. Additionally, the parameters given to the convention Dora originate from right estimations finished in our research center. Some reenactment is finished at the identical time as growing IPAT with every convention. Conference execution assessed thru sure insights and we center our examination across the ordinary power utilization of hubs, package deal deal conveyance proportion and dormancy in BS. Effects moreover everyday for diverse consequences recreations and $99 \%$ fact period in-between given. Normal manipulate usage is characterized as the percentage a number of the mixture sum of energy expended and the overall running time. The ordinary power usage implies located the middle price of for each hub and development is printed in decide 6 . Considering the fact that there's no responsibility cycle is characterised within the IEEE 802.15.4 conference, the everyday energy usage remains everyday while differing IPAT. The strength usage is reasonably high in moderate of steady tuning in to sit down. B-MAC offers higher consequences because it exploits obligation biking additives to spare power apparent contrasted and IEEE 802.15.Four As delineated in determine 6 , the convention Dora surpass splendid conventions in slight of the reality that the everyday strength usage is basically faded. The growth modified into essentially due to the inactive tuning in and catching the radio concealment inside the number one, which is enacted just at the same time as honestly important. This diagram moreover suggests that the convention DORA substantially an increasing number of affordable for WSN applications with low site traffic levels, but as but acting tremendous at the diploma of traffic is better.

From the above effects, it's miles furthermore practicable to parent the measure of energy placing some issue aside for the B-MAC and Do-Ra-contrasted and the IEEE 802.15.4 MAC. Given the among bundle deal appearance time of 15 seconds, B-MAC up97\% of energy sparing even as DoraMAC store as much as 99 .Ninety six \% of power, contrasted with the 802.15.Four MAC conference. That is the motive the everyday electricity utilization is plotted with a logarithmic scale in determine 6 , considering the ordinary strength utilization is 3 decrease request while the use of Dora contrasted with the B-MAC.

Package deal deal conveyance percentage is characterised as the quantity of parcels correctly got on the BS is separated by way of the quantity of bundles brought thru the organ. Radio bundle transmission is then given in discern 7 underneath various site traffic loads. Those results are consistent for IEEE 802.15.Four and Dora conference for package deal conveyance proportion is usually equal to as a minimum one, whilst it's far involved somewhere in the style of 0.90 five and 1 for B-MAC convention. The 
outcomes display the unwavering super our DoRaprotocol since almost every packets are correctly received.

Figure 6. Mean power consumption versus the inter packet arrival time for each protocol

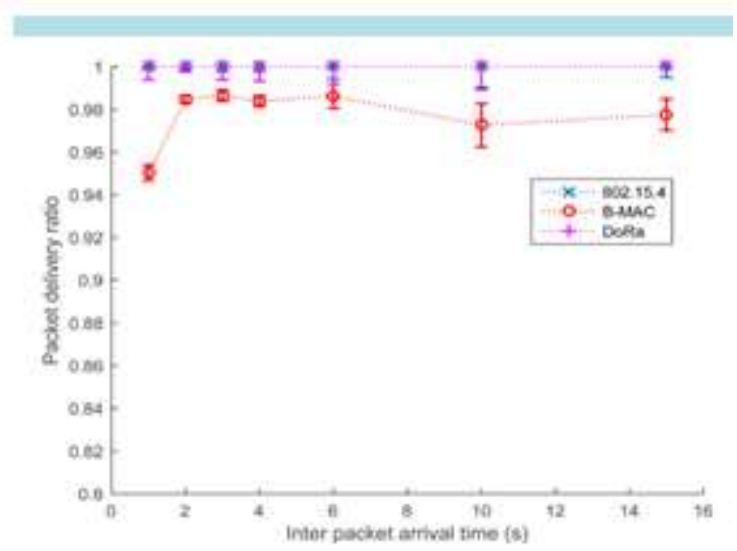

Figure 7. Packet delivery ratio versus the inter packet arrival time for each protocol

At that thing, the regular dormancy of every convention is portrayed in decide eight. For B-MAC and 802.15.4, the idleness is characterized due to the fact the distinction time among the package age on the hub and the parcel accumulating on the BS. For DoRa, the dormancy is characterized because the distinction time among the $\mathrm{WuC}$ age and the package deal gathering on the BS since the DoRa convention is a beneficiary commenced approach while the others are transmitter began techniques. The maximum minimal idleness is done by way of manner of the 802.15.Four convention because of its usually on radio, which allow to normally song in to the channel at the fee of better vitality utilization. B-MAC offers a reasonably high inertness blanketed someplace inside the range of 0.Five and $12 \mathrm{~d}$, essentially because of the lengthy prelude trying out and viable dispute issues. The idleness increments with the website site visitors charge as appeared in parent 8 considering conflict is positive to occur in better rush hour gridlock fee with B-MAC. The DoRa conference gives an kingdom of no interest marginally higher than 802.15.Four due to the lengthy $\mathrm{WuC}$ period. The idleness is usually equivalent to eleven. $7 \mathrm{~ms}$ regardless of the site visitors load, due to the fact there is no struggle while hubs are mainly awakening.

\subsection{DoRa drawback: The Overhearing problem}

Inactive tuning in and catching are absolutely smothered on the primary radio facet even as utilising a WuRx with commercial dressing capacities. On the WuRx aspect, there's no inert tuning in for the purpose that it is usually in relaxation mode except if a WuC triggers the WuRx. Anyways, catching nonetheless subsists on the WuRx side because each WuRx are activated while a $\mathrm{WuC}$ is despatched via way of the BS. This problem is especially number one whilst the WuRx is mentioned all of the time, which shows a low WuC entomb look time. The WuRx catching problem is often discarded inside the writing, as they bear in mind the WuR to be effective in low-website online visitors utility in which information are referred to from a few seconds to not often any hours. Be that as it can, catching may additionally likewise be an hassle if there should rise up an incidence of feasible outdoor impedances, for example, WiFi or Zigbee arranges inside the 2.Forty five Ghz band, which is probably super sufficient to trigger the WuRx. All subjects considered, the hub could remain in rest mode due to the fact no location might be identified yet a recognizable degree of strength could be gobbled due to the fact the WuRx could technique the obstruction as a WuC

Regardless, assessing the diploma of strength due to catching can display its impact on the overall vitality utilization and all of the more explicitly at the WuRx energy utilization. The hubs absolute power usage is given with the resource of the take a look at device however we must verify independently each phase cooperation. A clock is added to each MAC kingdom that allows you to gauge the span of each usa after reproduction. As we possibly am aware the strength con-sumption of every u . S ., we're then prepared to check the amount of strength wolfed by using way of every MAC nation. At that factor, the SDT version clarified in phase 3.1 is implemented for figuring the quantity of electricity wolfed due to catching. Following this model, overhearing occurs when there is a false alarm or a wake-up rejection after

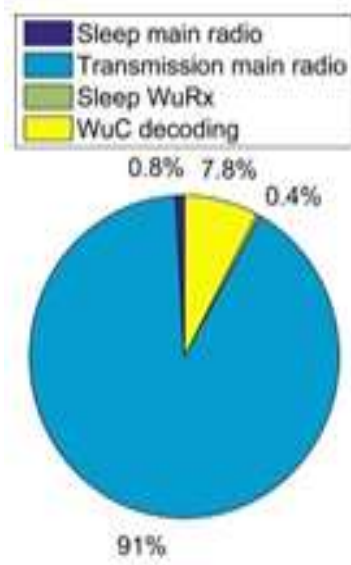

(a) Main radio and WuRx

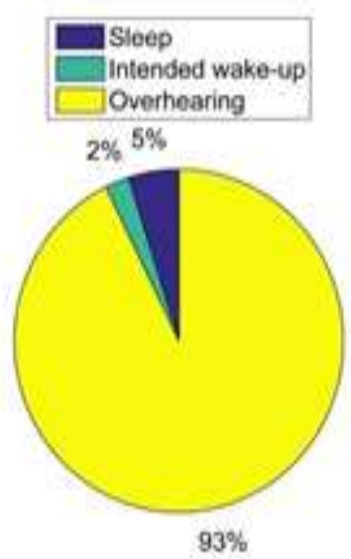

(b) WuRx only
Figure 8. Latency versus the inter packet arrival time for each protocol.

Detecting WUC. Each times are taken under consideration in the simulator and we derived strength for overhearing of possibilities obtained with the useful resource of simulation. The repartition the strength is given in determine nine for simulating the time among arrival WUC $50 \mathrm{~ms}$. On the equal time as thinking about each the precept radio and strength consumption WuRx in decide nine (a), ninety one $\%$ of the full energy consumption is because of the major radio transmission. Energy spent in sleep mode consumes simplest $1 \%$ of common energy intake at the same time as WUC decoding consumes eight\% of total strength consumption. Despite the fact that the primary radio cycle is lower than $1 \%$, the primary radio transmission with the most electricity client, because of this that maximum of the strength that is efficiently utilized by the protocol Dora. However, non-negligible quantity of 

WIRELESS SENSOR NETWORKS

energy used for interpreting WUC, led us to remember highquality WuRx energy repartition. As shown in decide nine (b), ninety three\% of the energy consumption is due overhears WuRx WUC meant for other nodes and only $2 \%$ of the overall power fed on for the purpose of waking up, due to this interpreting WUC supposed for the node. Then, repartitioning electricity WUC WuRx evaluated for differences in time between arrival and evolution are plotted in discern 10. Overhearing hassle is reduced at the same time as developing the time between arrival WUC, due to the fact that WuRx spend more

Figure 9. Energy repartition for the communication modules using DoRa protocol.

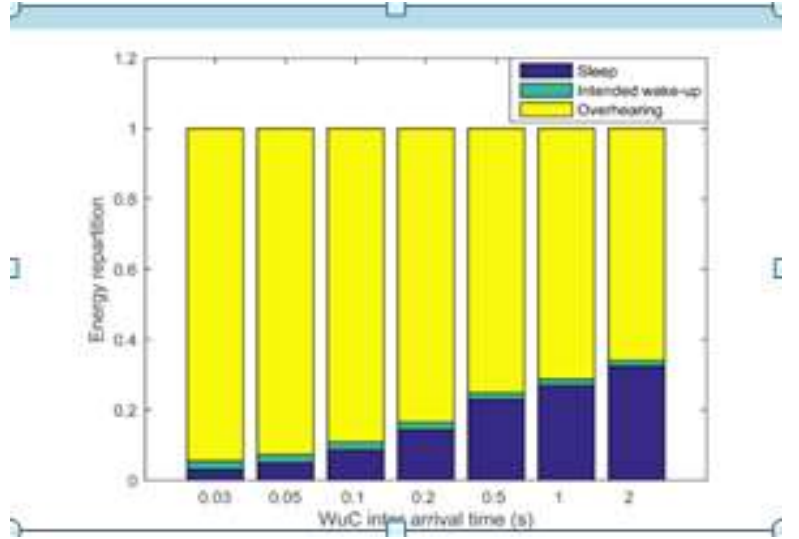

Figure 10. Energy repartition of the WuRx vs. WuC inter arrival time using Doa protocol

and more time in sleeping state. Nevertheless, overhearing still remains as the dominating source of energy consumption with the considered parameters. The results clearly show that the DoRa protocol suffers from an overhearing problem and a solution is proposed in the next section.

\section{DC-DORA PROTOCOL \& RESULTS}

\subsection{DC-DoRa Protocol Design}

This section portrays some other MAC conference proposed for settling the catching problem in wake-up radio situation. The convention is characterized as an augmentation of the DoRa convention, particularly the duty Cycled DoRa (DC-DoRa).As appeared with the aid of the DoRa correspondence rule in discern four, each hub way a $\mathrm{WuC}$ sent by way of way of the BS on account that every WuRx desires to pick out out whether or not the $\mathrm{WuC}$ is routed to the hub or now not. This $\mathrm{WuC}$ decoding stage ocpuppies increasingly more habitually whilst we increment the amount of hubs or lessening the $\mathrm{WuC}$ entomb appearance time, as regarded in the past phase. We endorse to lower or perhaps expel this catching problem via debilitating the WuRx usefulness for a selected time frame, which starts offevolved soon after the hub has despatched facts to the BS. All through this period, the hub won't have been given any $\mathrm{WuC}$ because the $\mathrm{BS}$ wants to call for records from distinctive hubs.

We constitute the spherical duration Trp because the difference time amongst each datum call for from a comparable hub much less $1 / 2$ span of the break window
Tout, with its demeanor given in Equation (four). This definition empowers the WuRx usefulness with an adequate length in advance than being mentioned via the BS, so as to live away from any clock waft trouble.

The spherical duration Trp is decided thru the BS and imparted to the hubs by means of using incorporating its incentive into the $\mathrm{WuC}$ message, after the purpose MAC deal with vicinity as delineated in figure eleven. The spherical duration may additionally need to likewise be set into the hub earlier than the arrangement, however this method is preferred for its adaptability since the spherical duration may be adjusted through the WSN application.

The present day correspondence favored using the DCDoRa conference is portrayed in discern 12, demonstrating that each hub forms just the WuC consider to itself Catching from $\mathrm{WuC}$ predicted to at least one-of-a-type hubs may be absolutely stifled with this proposed device. Regarding the all of the way right down to earth utilization practicality, the Do-Ra hub configuration displayed in phase 2.1 empowers such machine. The WuRx usefulness can absolutely be in brief incapacitated via the MCU with a sign on top of things. The MCU is then placed into profound rest mode with the profound rest protect canine clock (DSWDT) taking walks, so that you can rest for the duration of the spherical length. In the wake of awakening, the MCU empowers the WuRx usefulness and returns to profound rest once more with out the DSWDT on foot. Walking the DSWDT likewise expends some greater strength in profound relaxation mode, that is considered in the reenactment by way of the use of considering various sound asleep states.

\subsection{DC-DoRa overall performance evaluation}

On this section, the DC-DoRa execution is classed and contrasted with the DoRa convention. A comparable tool portrayed in segment 3 is mimicked utilizing the DC-DoRa convention the usage of parameters given in desk 2 . The principle contrasts in parameters between each convention are the prevailing utilization in resting nation and the $\mathrm{WuC}$ length, each being especially progressed with the DC-DoRa conference.

The energy repartition is displayed in determine 13 for reenactment outcomes with a $\mathrm{WuC}$ entomb look time of 50 ms. As appeared in figure thirteen(a), the $\mathrm{WuC}$ decoding just speaks to below $1 \%$ of the all out energy usage, contrasted with the beyond $8 \%$ of the DoRa convention. Transmission with fundamental radio speaks to $97 \%$ of the total energy consumption, which means that the WuRx energy consumption is very negligible compared to the main radio energy consumption.

Figure 11. Communication principle with the DCDoRa protocol. 


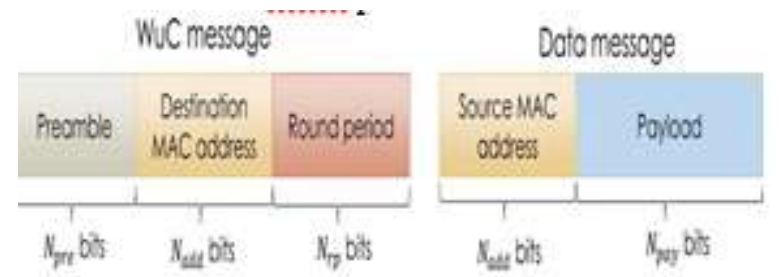

Figure 12. Frame structure of exchanged messages between the BS and nodes with DC-DoRa protocol.

Significant increment may be evaluated in figure 13 (b) to repartition WuRx power. Catches essentially killed by means of the DC-Dora convention because it clearly speaks to one $\%$ of the power usage. The principle power consumer is the rest mode with $87 \%$ of the power utilization at some point of interpreting implied waking just talk to eleven\%. The WuRx repartition strength is then plotted while differing the time amongst appearance WUC parent 14. Vitality usage for relaxation states greater noteworthy addition even as expanding WUC time among look and spoke to nearly the totality of the power utilization sooner or later of the time amongst look WUC is equal 2 seconds.

Assessing the power repartition provide a outstanding comprehension of what need to be finished to beautify electricity productiveness, specially it indicates that DCDora convention appears after the catching difficulty truely. Anyways, do no longer supply a lower of the energy provided by the new protocol neither the amount of energy consumed by the

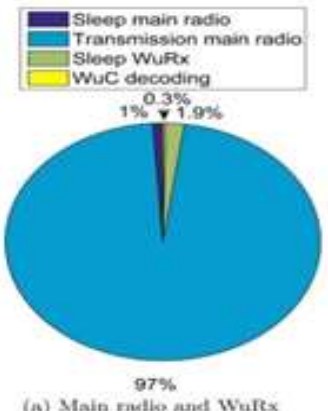

(a) Main raetlo and Wufex

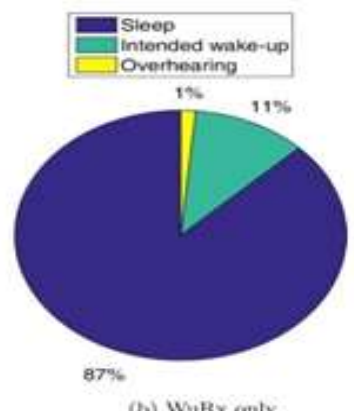

(b) WaRx enty
Figure 13. Energy repartition for the communication modules using DC-DoRa protocol.

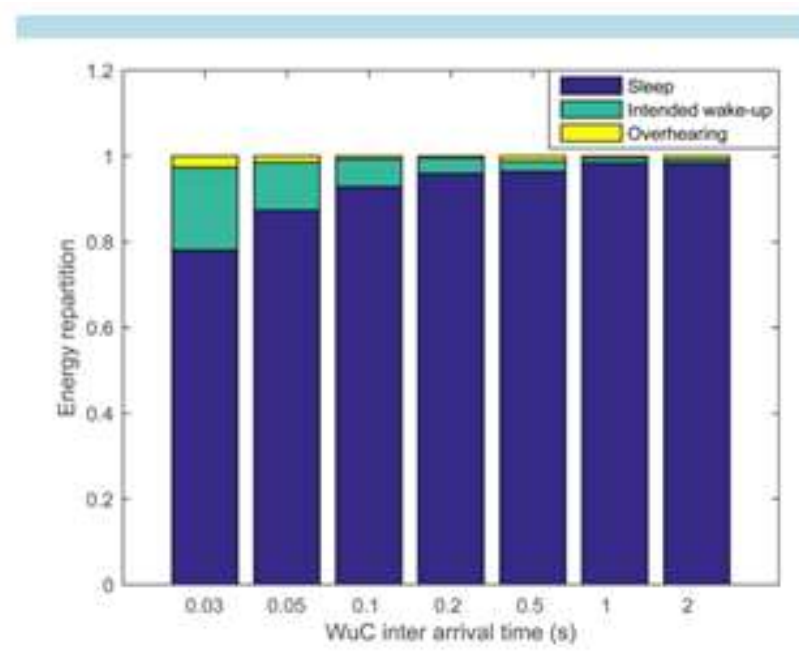

Figure 14. Energy repartition of the WuRx vs. WuC inter arrival time using DC-DoRa protocol

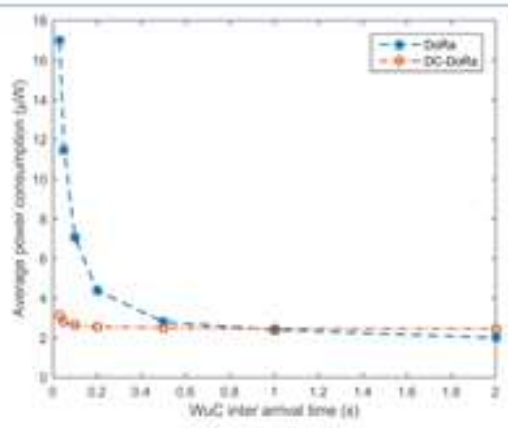

Figure 15. WuRx average power consumption vs. WuC inter arrival time using DC-DoRa protocol.

Framework. The WuRx regular power utilization is then plotted rather than the $\mathrm{WuC}$ bury look time in discern 15 making use of the DoRa or DC-DoRa conference. The consequences show the energy effectiveness of the DCDoRa convention com-pared to the DoRa conference for low WuC bury appearance time, wherein the catching problem is steadily articulated. For WuC entomb look time better than $12 \mathrm{nd}$, the DoRa convention is extra energy proficient while you don't forget that hubs invest more frequently than now not in rest mode, and the DC-DoRa slumbering kingdom expends extra power than the DoRa napping country because of the DSWDT clock running in DC-DoRa. Furthermore, the WuRx ordinary power utilization is isolated via manner of up to five for a $\mathrm{WuC}$ entomb appearance time of $30 \mathrm{~ms}$.For event, the DoRa conference is affordable for WSN software together with a slight physical marvels moni-toring. Anyhow, the DC-DoRa conference performs better at the same time as the bodily wonders variety is well underneath the second.

\section{CONCLUSION AND PERSPECTIVE}

The DoRa convention extensively diminishes the hub's capability utilization contrasted with IEEE 802.15.Four and B-MAC. Accordingly, the semi-inactive layout of WuR spares a outstanding deal of strength contrasted with the method constantly in radio or twofold cycle mode. A restrict of the WuR frameworks is the overheading of $\mathrm{WuC}$ for precise hubs, which converts into in addition strength usage thru the standard WuR convention or maybe the DoRa conference. The DC-DoRa conference turned into then proposed to attend to this tough problem, which contains of a jogging cycle component within the $\mathrm{WuR}$ framework. The consequences glaringly display off the viability of the conference due to the fact the issue of over-paying attention to is kind of killed. In this manner, the vitality usage of the WuRx is exceedingly reduced with the DC-DoRa for the incessant gathering of the $\mathrm{WuC}$, whilst the DoRa convention is preferred for a greater prolonged look time than the $\mathrm{WuC}$

In future works, DoRa and DC-DoRa conventions are joined together and may be carried out to get superior WSN workout scenario, mainly with the concept of a flexible sink arew collected in diverse places. We're furthermore at present actualizing those conventions in exploratory case with our WuRx models. 

WIRELESS SENSOR NETWORKS

\section{REFERENCES}

1. Borgia, E. (2014) The net of things imaginative and prescient: Key talents, packages and Open troubles. Pc Communications, fifty 4, 1-31. Http://dx.Doi.Org/10.1016/j.Comcom.2014.09.008

2. Anastasi, G., Conti, M., Di Francesco, M. What's greater, Passarella, A. (2009) electricity Conservation in wi-fi Sensor Networks: A Survey. Impromptu Networks, 7, 537-568.Http://dx.Doi.Org/10.1016/j.Adhoc.2008.06.003

3. Rault, T., Bouabdallah, A. What's extra, Challal, Y. (2014) energy performance in wireless Sensor Networks: A top-Down Sur-vey. Computer Networks, 67, 104-122. Http://dx.Doi.Org/10.1016/j.Comnet.2014.03.027

4. Adhikari, R. (2014) A Meticulous study of diverse Medium get access to govern Protocols for wi-fi Sensor Networks journal of network and laptop applications, forty one, 488-504.

5. Polastre, J., Hill, J. What's greater, Culler, D. (2004) flexible Low energy Media get right of entry to for wireless Sensor Networks. Hold ings of the second one global convention on Embedded Networked Sensor systems, Baltimore, 3-five November 2004, 90 5-107. Http://dx.Doi.Org/10.1145/1031495.1031508

6. Ye, W., Heidemann, J. What's greater, Estrin, D. (2002) An power-inexperienced MAC Protocol for wi-fi Sensor Networks. INFOCOM Twenty-First Annual Joint convention of the IEEE computer and Communications Societies, the huge apple, 23-27 June 2002, 1567-1576.

7. Gu, L. What's more, Stankovic, J.A. (2004) Radioinduced Wake-Up functionality for Sensor Networks. IEEE actual-Time and Embedded generation and applications Symposium, Toronto, 25-28 may also additionally 2004, 27-37.

8. Oller, J., Demirkol, I., Casademont, J., Paradells, J., Gamm, G.U. What's extra, Reindl, L. (2013) overall performance evaluation and Comparative analysis of SubCarrier Modulation Wake-Up Radio systems for energy-green wi-fi Sensor net-works. Sensors, 14, 22-51. Http://dx.Doi.Org/10.3390/s140100022

9. Sthapit, P. What is extra, Pyun, J.- Y. (2011) results of Radio prompted Sensor MAC Protocol over wireless Sensor network. Eleventh IEEE global convention on pc and data era, Paphos, 31 August-2 September 2011, 546551. Http://dx.Doi.Org/10.1109/cit.2011.58

10. Ameen, M.A., Niamat, U., Sanaullah, C.M. What's extra, Kyungsup, k. (2011) A MAC Protocol for body place Networks the usage of Out-of-Band Radio. Remote convention 2011-Sustainable wi-fi technologies (european wireless), eleventh Euro-pean, Vienna, 27-29 April 2011, 1-6.

11. Ramachandran, V.Okay., Zwaag, B.J., Meratnia, N. What's more, Havinga P.J.M. (2014) assessment of MAC Protocols with Wake-Up Radio for Implantable frame Sensor Networks. Fourth international conference on decided on subjects in Mo-bile and wireless Networking (MoWNet2014), Rome, eight-10 September 2014, 173 one hundred eighty. Http://dx.Doi.Org/10.1016/j.Procs.2014.12.1/2

12. Oller, J., Demirkol, I., Casademont, J., Paradells, J., Gamm, G.U. Additionally, Reindl, L. (2016) Has Time Come to update From obligation-Cycled MAC Protocols to Wake-Up Radio for wi-fi Sensor Networks? IEEE/ACM Transactions on Networking, 24, 674-687. Http://dx.Doi.Org/10.1109/TNET.2014.2387314

13. Varga, A. (2001) The OMNeT++ Discrete occasion Simulation machine. Tactics of the ecu Simulation Multiassembly (ESM2001), Prague, 6-9 June 2001, 1-7.

14. Kopke, A., Swigulski, M., Wessel, ok., Willkomm, D., Haneveld, P.T., Parker, T.E.V., Visser, O.W., Lichte, H.S. Moreover, Valentin, S. (2008) Simulating wi-fi and cell Networks in OMNeT++ the MiXiM imaginative and prescient. Methods of the first global conference on Simulation equipment and strategies for Communications, Networks and structures and Workshops, Marseille, three-7 March 2008, 1-eight. Http://dx.Doi.Org/10.4108/icst.Simutools2008.3031

15. Lebreton, J. Moreover, Murad, N. (2015) Implementation of a Wake-Up Radio move-Layer Protocol in OMNeT++/MiXiM. Tactics of the second one OMNeT++ network Summit, Zurich, three-4 September $2015,1-5$

16. Jelicic, V., Magno, M., Brunelli, D., Bilas, V. Additionally, Benini, L. (2012) Analytic contrast of Wake-Up Receivers for Wsns and benefits over the Wake-On Radio Scheme. Methods of the seventh ACM Workshop on common performance Moni-toring and size of Heterogeneous wi-fi and stressed out Networks, Paphos, 21-25 October 2012, 99-106. Http://dx.Doi.Org/10.1145/2387191.2387206

17. Ansari, J., Pankin, D. Also, Mahonen, P. (2009) Radioprecipitated Wake-americawith Addressing talents for notably Low energy Sensor community programs. International magazine of wi-fi information Networks, sixteen, 118-one hundred thirty. Http://dx.Doi.Org/10.1007/s10776-009-0100-6

18. Spenza, D., Magno, M., Basagni, S., Benini, L., Paoli, M. Additionally, Petrioli, C. (2015) past responsibility biking: Wake-Up Ra-dio with Selective Awakenings for lengthy-Lived wireless Sensing systems. 2015 IEEE conference on laptop Communications (INFOCOM), Kowloon, 26 April-1 may also additionally 2015, 522530 .

Http://dx.Doi.Org/10.1109/INFOCOM.2015.7218419

19. Lebreton, J., Murad, N., Kandukuri, S. Also, Lorion, R. (2016) An strength-green Addressing Mechanism for Wake-Up Radio systems. 2016 wi-fi Telecommunications Symposium (WTS), London, 18-20 April 2016, 1-6.

20. Abdi, A. (2007) signal Detection idea (SDT). In: Salkind, N., Ed., Encyclopedia of measurement and facts, Sage, Thousand thoroughly, 886-889.

21. IEEE pc Society (2011) IEEE cutting-edge for neighborhood and Metropolitan region Networks. Element 15.4, LAN/man necessities Committee. 